\title{
The Baikal neutrino telescope-Results and plans
}

\author{
A. Avrorin ${ }^{\mathrm{a}}, \mathrm{V}$. Aynutdinov ${ }^{\mathrm{a}}, \mathrm{V}$. Balkanov ${ }^{\mathrm{a}}, \mathrm{I}$. Belolaptikov ${ }^{\mathrm{d}}, \mathrm{D}$. Bogorodsky ${ }^{\mathrm{b}}, \mathrm{N}$. Budnev $^{\mathrm{b}}$,

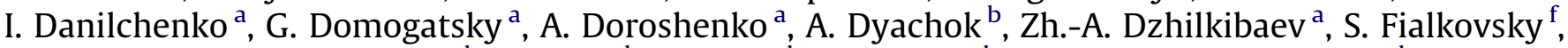 \\ O. Gaponenko a , K. Golubkov ${ }^{\text {d }}$, O. Gress ${ }^{\text {b }}$, T. Gress ${ }^{b}$, O. Grishin ${ }^{b}$, A. Klabukov ${ }^{\text {a }}$, A. Klimov ${ }^{\text {, }}$, \\ A. Kochanov ${ }^{\text {b }}$, K. Konischev ${ }^{\text {d }}$, A. Korobchenko ${ }^{b}$, A. Koshechkin ${ }^{\text {a }}$, V. Kulepov ${ }^{f}$, D. Kuleshov ${ }^{\text {a }}$,

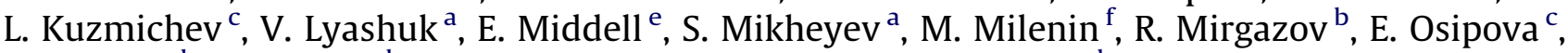 \\ G. Pan'kov ${ }^{b}$, L. Pan'kov ${ }^{b}$, A. Panfilov ${ }^{a}$, D. Petukhov ${ }^{a}$, E. Pliskovsky ${ }^{\text {, }}$, P. Pokhil ${ }^{a}$, V. Poleschuk ${ }^{a}$,

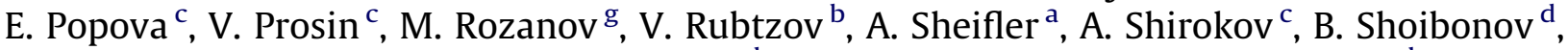 \\ Ch. Spiering ${ }^{\text {e }}$ O. Suvorova ${ }^{\text {a }}$, B. Tarashansky ${ }^{\text {b }}$, R. Wischnewski ${ }^{\text {e,* }}$, A. Zagorodnikov $^{\text {b }}$, V. Zhukov $^{\text {a }}$ \\ a Institute for Nuclear Research, 60th October Anniversary pr. 7a, Moscow 117312, Russia \\ ${ }^{\mathrm{b}}$ Irkutsk State University, Irkutsk, Russia \\ c Skobeltsyn Institute of Nuclear Physics MSU, Moscow, Russia \\ d Joint Institute for Nuclear Research, Dubna, Russia \\ e DESY, Zeuthen, Germany \\ ${ }^{f}$ Nizhni Novgorod State Technical University, Nizhni Novgorod, Russia \\ ${ }^{g}$ St.Petersburg State Marine University, St.Petersburg, Russia \\ ${ }^{\mathrm{h}}$ Kurchatov Institute, Moscow, Russia
}

\section{A R T I C L E I N F O}

Available online 11 June 2010

Keywords:

Neutrino telescopes

Neutrino astronomy

UHE neutrinos

BAIKAL

\begin{abstract}
A B S T R A C T
The Neutrino Telescope NT200 is operated since 1998 and was upgraded to the 10 Mton detector $\mathrm{NT200+}$ in 2005. The preparation towards a $\mathrm{km}^{3}$-scale (Gigaton Volume) detector in Lake Baikal is currently a central activity. As an important milestone, a $\mathrm{km}^{3}$-prototype string, based on completely new technology, has been installed and was operating together with NT200+ since April 2008. Also selected astroparticle physics results from the long-term operation of NT200 are presented.
\end{abstract}

(c) 2010 Elsevier B.V. All rights reserved.

\section{Introduction}

The Baikal Neutrino Telescope NT200 is operating in Lake Baikal at a depth of $1.1 \mathrm{~km}$ and is taking data since 1998. Since 2005, the upgraded 10-Mton scale detector NT200+ is in operation. Detector configuration and performance have been described elsewhere $[1,2]$. The most recent milestone of the ongoing $\mathrm{km}^{3}$-telescope research and development work (R\&D) was the installation of a "new technology" prototype string in spring 2008, as a part of NT200+. Fig. 1 gives a sketch of the current status of the telescope NT200+, including the $\mathrm{km}^{3}$-prototype string.

In this paper we review selected astroparticle physics results from long-term operation of NT200, in particular, an improved limit on a diffuse astrophysical neutrino flux and upper limits on the muon flux from annihilations of hypothetical weakly interacting massive particles (WIMPs) in the Earth and the Sun. We also discuss the R\&D activities towards a $\mathrm{km}^{3}$-scale Baikal

\footnotetext{
* Corresponding author.

E-mail address: ralf.wischnewski@desy.de (R. Wischnewski).
}

telescope. Other physics results (atmospheric neutrinos, flux limits for relativistic magnetic monopoles and high-energy atmospheric muons), new acoustic neutrino detection technology tests and related science (limnology) can be found in Ref. [3].

\section{Selected physics results from NT200}

\subsection{A search for neutrinos from WIMPs in the Earth and in the Sun}

A possible signal from dark matter WIMP annihilations in the Earth and in the Sun would reveal as an excess of upward going muons over atmospheric neutrinos arriving either from near vertical or from the direction of the Sun, respectively. We have used the experimental data of NT200 taken between April, 1998 and March, 2003. In case of the Earth signal, event selection relies on a series of quality cuts which are tailored to the response of the telescope to nearly vertically upward going muons. The energy threshold is about $E_{\text {thr }} \sim 10 \mathrm{GeV}$ in this analysis. We have selected 48 neutrino events for 1038 live days, compared to 56.6 events expected from atmospheric neutrinos with oscillation parameters 


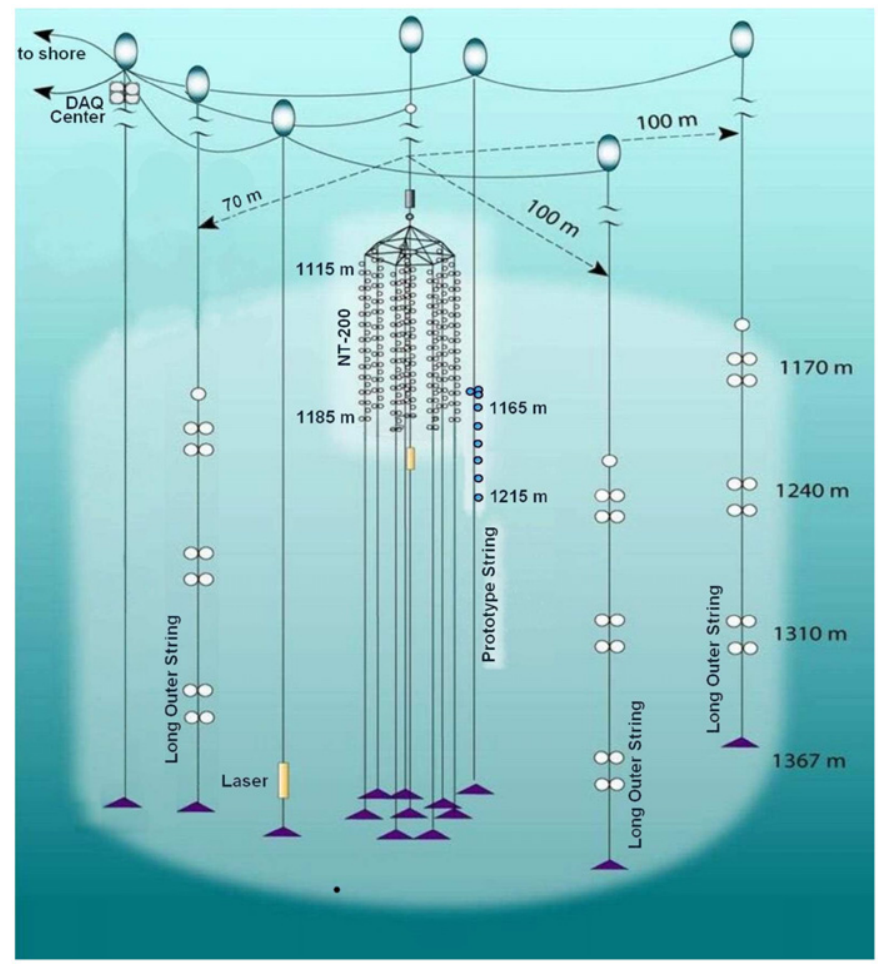

Fig. 1. The Baikal Telescope NT200+ as of 2008: the compact NT200 (center), 3 long outer strings and the new technology $\mathrm{km}^{3}$-prototype string.

of Super-Kamiokande results [4], and 73.1 events without oscillations. With no evidence for an excess above the atmospheric neutrino expectation, the upper limit at $90 \%$ confidence level (c.l.) on the muon flux from the center of the Earth was determined as $F<3.7 \times 10^{-15} \mathrm{~cm}^{-2} \mathrm{~s}^{-1}$ (for WIMP masses greater than $100 \mathrm{GeV}$, and normalized to $\left.E_{t h r}=1 \mathrm{GeV}\right)$.

In case of the Sun we have applied two sorts of quality cuts, optimized for high and low masses [5]. We have selected 510 and 2376 upward going muon candidates in the two data samples for 1007 live days. For both samples the distributions of correlation angles between these muons and the Sun were compared to the corresponding off-source background expectation. No indication for excess muons were found.

Upper limits on muon fluxes from the Sun obtained by neutrino telescopes are shown in Fig. 2 (adapted from Ref. [6], all limits are for $1 \mathrm{GeV}$ threshold). The Baikal curve corresponds to hard neutrino spectra, see Ref. [5] (also for AMANDA-II and IceCube values). The presented results are preliminary, and allow to estimate the NT200 sensitivity for high energy neutrinos from DM annihilation processes in the Sun.

\subsection{A search for extraterrestrial high-energy neutrinos}

The BAIKAL survey for high energy neutrinos searches for bright cascades produced at the neutrino interaction vertex in a large volume around the telescope. A full cascade reconstruction algorithm (for vertex, direction, energy) was applied to the data [11]. Cuts were then placed on this reconstructed cascade energy to select neutrino events. The reconstructed energy distribution of data is shown in Fig. 3 (dots). Eight events were reconstructed as upward going cascades (zenith angle $\theta>90^{\circ}$, distribution in dashed box in Fig. 3). Also the MC-generated (histogram) and reconstructed (boxes) energy distributions from simulated atmospheric muons are shown in Fig. 3; 12 upward reconstructed cascade-like events are expected. As seen from Fig. 3, within

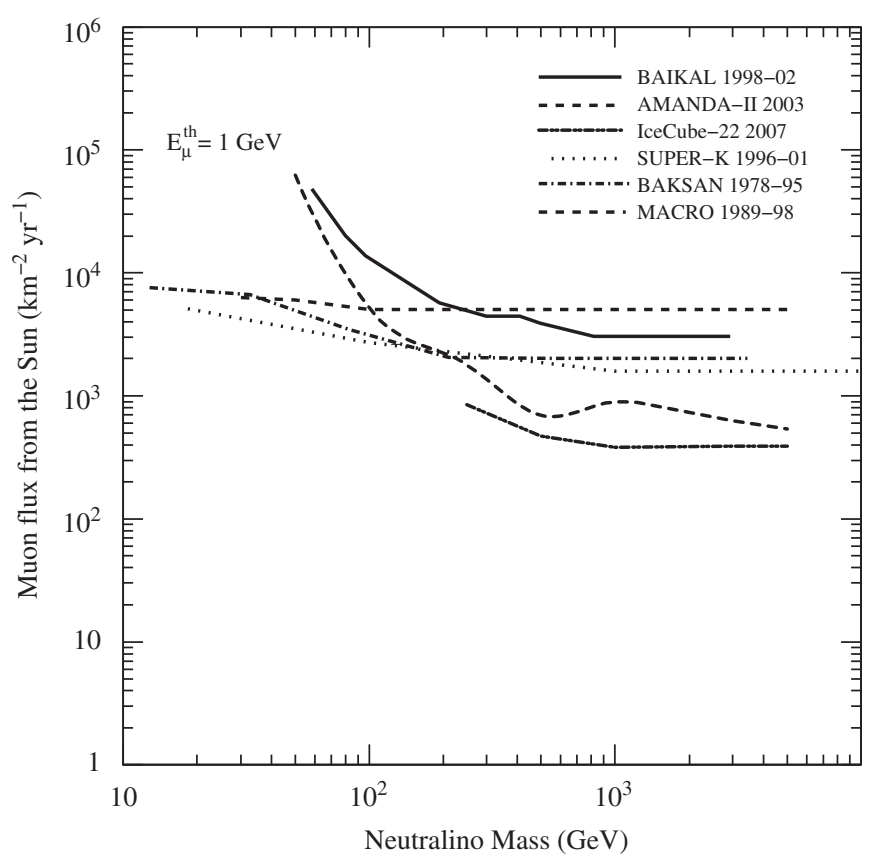

Fig. 2. Upper limits at $90 \%$ c.l. on the muon flux from the Sun versus WIMP mass: Baikal NT200, MACRO [7], Baksan [8], Super-Kamiokande [9], AMANDA-II [6] and IceCube-22 [10].

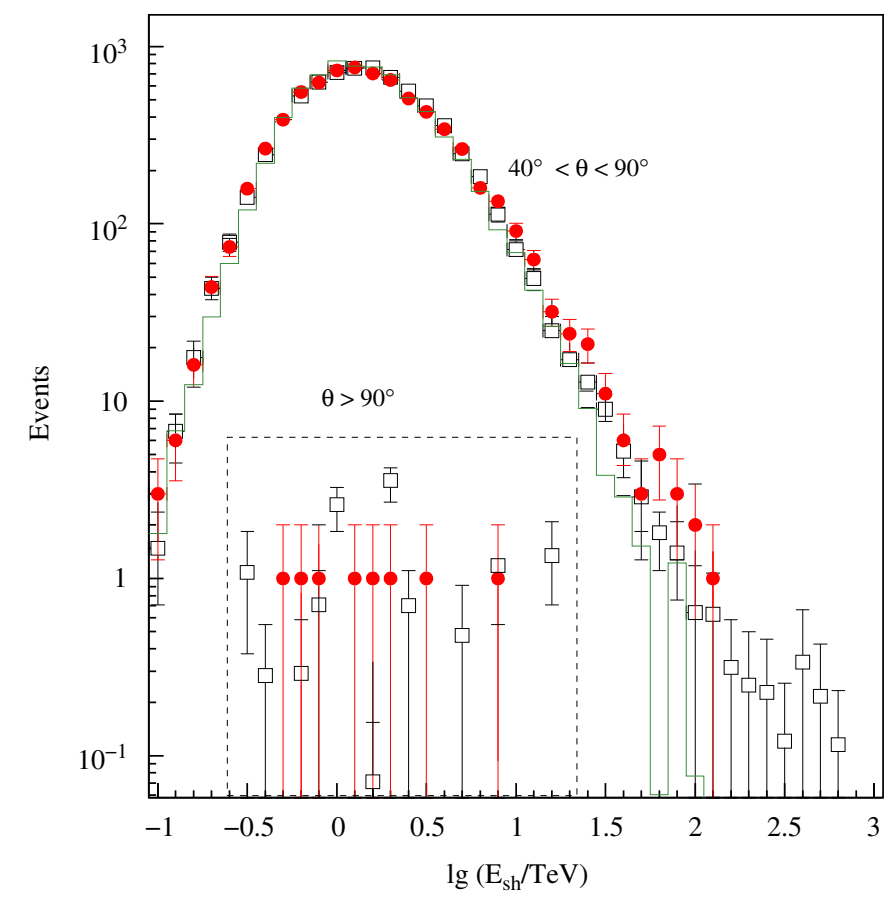

Fig. 3. Reconstructed cascade energy distribution for data (full dots) and for MC-generated atmospheric muons (boxes); true MC energy distribution given as histogram.

systematic and statistical uncertainties there is no significant excess above the background from atmospheric muons. We introduce the following final neutrino signal cuts on the cascade energy: $E_{s h}>130 \mathrm{TeV}$ and $E_{s h}>10 \mathrm{TeV}$ for downward and upward going cascades, respectively. With zero observed events and $2.3 \pm 1.2$ expected background events, a 90\% confidence level upper limit on the number of signal events of $n_{90 \%}=2.4$ is 


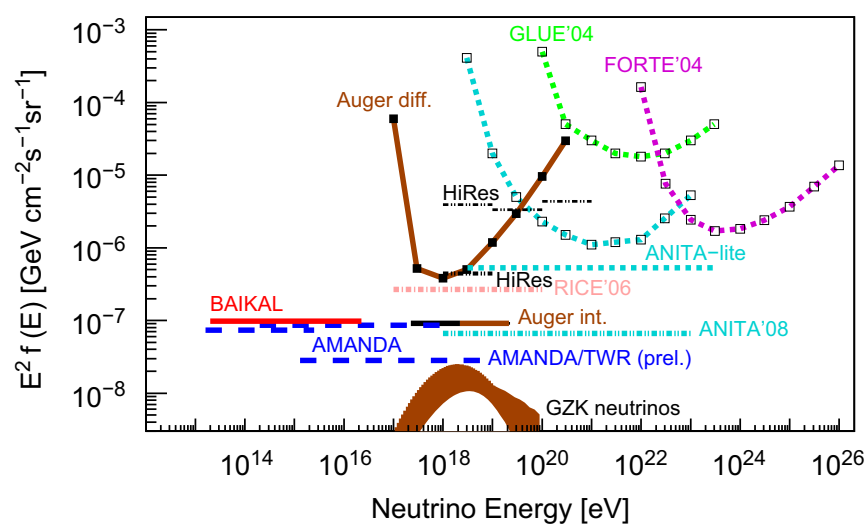

Fig. 4. Experimental limits (90\%CL) on the diffuse extraterrestrial flux of high energy neutrinos, from Baikal-NT200 (this work) and other experiments (see text). Differential and integral $\left(E^{-2}\right)$ limits are shown, the flux is normalized per $v$-flavor).

obtained. For an $E^{-2}$ behaviour of the neutrino spectrum and a flavor ratio $v_{e}: v_{\mu}: v_{\tau}=1: 1: 1$, the $90 \%$ C.L. upper limit on the neutrino flux of all flavors obtained with the Baikal neutrino telescope NT200 is: $E^{2} \Phi<2.9 \times 10^{-7} \mathrm{~cm}^{-2} \mathrm{~s}^{-1} \mathrm{sr}^{-1} \mathrm{GeV}$, for $20 \mathrm{TeV}<E_{v}<20 \mathrm{PeV}$. We note, that this new NT200 result is a significant improvement of the earlier obtained limit [12].

In Fig. 4 we compare 90\% CL limits on diffuse UHE neutrino fluxes from NT200 and other experiments (figure adapted from Ref. [13]). The experimental flux limits are given for a single flavor (i.e. all-flavor results are scaled by $1 / 3$ ), and in both differential and integral (for an $E^{-2}$ spectrum) form. Limits are from AMANDA [14,15], AMANDA-TWR [16], Auger [13], HiRes [17], GLUE [18], FORTE [19], RICE [20], ANITA-lite [21] and ANITA [22].

\section{Towards a $\mathbf{k m}^{3}$-scale detector in Lake Baikal}

The Baikal collaboration follows since several years a R\&D program for a $\mathrm{km}^{3}$-scale neutrino telescope in Lake Baikal. The Baikal $\mathrm{km}^{3}$-detector will have a relatively flexible structure, which allows for a rearrangement of the main building blocks (clusters), to adapt for requirements of new scientific goals, if necessary. Fig. 5 shows the baseline design of the Baikal $\mathrm{km}^{3}$-detector. The total number of 2304 optical modules (OMs) will be arranged at 96 strings with 24 OMs each, and an instrumented length of $350-460 \mathrm{~m}$. Interstring distances will be $60-120 \mathrm{~m}$. The strings are grouped in 12 clusters which will form independent arrays. A total volume of $0.4-0.6 \mathrm{~km}^{3}$ will be instrumented with photo-sensors. The effective area for muons above $10 \mathrm{TeV}$ with an angular resolution of $0.5^{\circ}-1^{\circ}$ is $0.2-0.5 \mathrm{~km}^{2}$, and the effective volume for cascade events above $100 \mathrm{TeV}$ with angular resolution $2^{\circ}-4^{\circ}$ is $0.15-0.7 \mathrm{~km}^{3}$.

The construction of $\mathrm{NT200}+$ was a first step towards a $\mathrm{km}^{3}$-scale neutrino telescope. NT200+ is a natural laboratory to verify many key elements and design principles of the new telescope. The most recent $\mathrm{km}^{3}$-milestone was the construction and installation of a new technology prototype string in spring 2008 and its upgrade in 2009. The $\mathrm{km}^{3}$-prototype string consists of 12 new generation optical modules with 12 in./13 in. photomultipliers XP1807 (Photonis) and R8055 (Hamamatsu). The basic goals of this prototype string are: in-situ test of basic elements of the future detector (new optical modules, DAQ system and cabling system); studies of the basic triggering approach for the $\mathrm{km}^{3}$-detector; comparison of the classical TDC-based approach with a FADC-based full pulse shape PMT readout. Prototype string design and first results are described in detail in Ref. [23], calibration and verification tests have been successful.

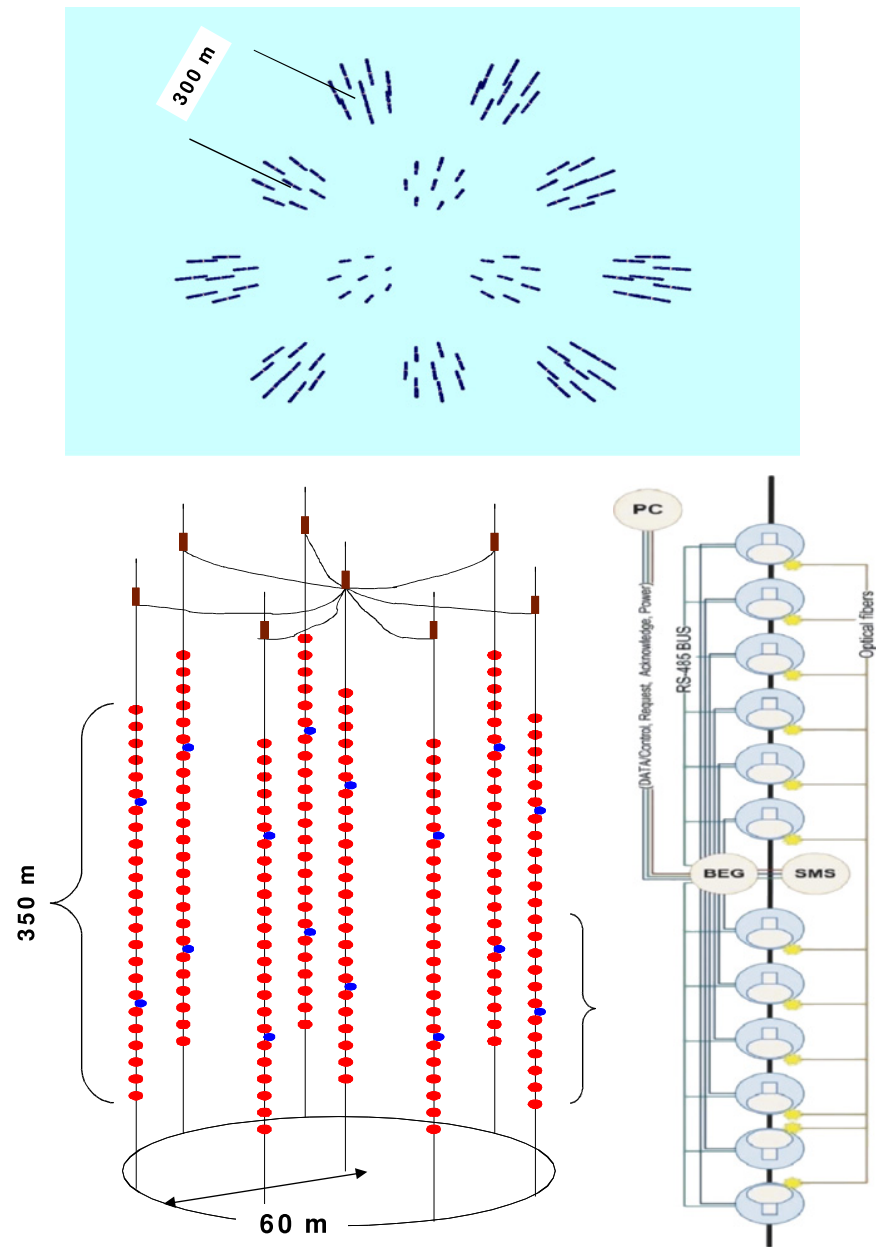

Fig. 5. Layout of the planned Baikal $\mathrm{km}^{3}$-scale neutrino detector (Gigaton Volume Detector). Upper panel: top view of the 12 clusters (2304 OMs); Lower left panel: layout of a cluster with 8 strings (192 OMs, 24 OMs per string); Lower right panel: a half-string, consisting of 12 OMs.

\section{Conclusion}

The Baikal neutrino telescope NT200 is working since April 1998. On the road towards a $\mathrm{km}^{3}$-scale neutrino telescope in Lake Baikal, significant upgrades of the detector have been done in spring 2005. Up to now the NT200+ telescope of 5 Mton enclosed volume is in operation, together with a $\mathrm{km}^{3}$-prototype string installed/upgraded in spring 2008/09. The technical design report for the $\mathrm{km}^{3}$-scale Baikal detector will be finished in 2011 .

An analysis of the NT200 data samples for the 1998-2002 data taking seasons has been carried out. With an improved method, based on the reconstructed cascade energy, a significantly lowered upper limit for a diffuse astrophysical $\left(v_{e}+v_{\mu}+v_{\tau}\right) E^{-2}$ flux has been obtained. The same data samples were analyzed for neutrinos from WIMP annihilation in both the Sun and the Earth. No excess signals were found, therefore upper limits on an additional muon flux from the Sun and the Earth in dependence on the WIMP mass have been set.

\section{Acknowledgements}

This work was supported in part by the Russian Ministry of Education and Science, by the German Ministry of Education and Research, by the Russian Found for Basic Research (Grants 08-0200432-a, 09-02-10012-k, 07-02-00791, 08-02-00198, 09-02-10001-k, 
09-02-00623-a, 09-02-12295), by the Grant of the President of Russia NSh-321.2008-2, by the program "Development of Scientific Potential in Higher Schools" (projects 2.2.1.1/1483, 2.1.1/1539, 2.2.1.1/5901), and by NATO-Grant NIG-9811707(2005).

\section{References}

[1] V. Aynutdinov, et al., Nucl. Instr. and Meth. A 588 (2008) 99.

[2] V. Aynutdinov, et al., Nucl. Instr. and Meth. A 567 (2006) 433

[3] V. Aynutdinov, et al., Nucl. Instr. and Meth. A 602 (2009) 14.

[4] Y. Fukuda, et al., Phys. Rev. Lett. 81 (1998) 1562.

[5] A. Avrorin, et al., in: Proceedings of 31st ICRC, icrc1165, Lodz, 2009, and arXiv:0909.5589.

[6] C. De Clerq, et al., in: Proceedings of IDM2008, Stockholm, 2008.

[7] M. Ambrosio, et al., Phys. Rev. D 60 (1999) 082002.
[8] M.M. Boliev, et al., Nucl. Phys. (Proc. Suppl.) 48 (1996) 83;

M.M. Boliev, et al., in: Dark Matter in Astro- and Particle Physics, vol. 711, 1997; O.V. Suvorova, hep-ph/9911415, 1999.

[9] S. Desai, et al., Phys. Rev. D 70 (2004) 083523.

[10] R. Abbasi, et al., Phys. Rev. Lett. 102 (2009) 201302.

[11] A. Avrorin, et al., Astron. Lett. 35 (2009) 651.

[12] V. Aynutdinov, et al., Astropart. Phys. 25 (2006) 140

[13] J. Abraham, et al., Phys. Rev. D 79 (2009) 102001.

[14] A. Achterberg, et al., Phys. Rev. D 76 (2007) 042008

[15] M. Ackermann, et al., Astrophys. J. 675 (2008) 1014.

[16] A. Silvestri, et al., in: Proceedings of 31st ICRC, icrc0546, Lodz, 2009.

[17] K. Martens, et al., arXiv:0707.4417;

R.U. Abbasi, et al., arXiv:0803.0554

[18] P.W. Gorham, et al., Phys. Rev. Lett. 93 (2004) 041101.

[19] N.G. Lehtinen, et al., Phys. Rev. D 69 (2004) 013008.

[20] I. Kravchenko, et al., Phys. Rev. D 73 (2006) 082002.

[21] S.W. Barwick, et al., Phys. Rev. Lett. 96 (2006) 171101.

[22] P.W. Gorham, et al., arXiv:0812.2715.

[23] V. Aynutdinov, et al., Nucl. Instr. and Meth. A 602 (2009) 227. 